\title{
Maxima of the spectral reflectance ratio of polarized waves used to measure the thickness of a nonabsorbing thin film
}

\author{
P. Hlubina* , J. Luňáček, D. Ciprian \\ Department of Physics, Technical University Ostrava, 17. listopadu 15, \\ 70833 Ostrava-Poruba, Czech Republic
}

\begin{abstract}
A simple method to determine the thickness of a nonabsorbing thin film on an absorbing substrate from maxima of the ratio between the spectral reflectances of $p$ and $s$-polarized components reflected from the thin-film structure is presented. The spectral reflectance ratio, which can be measured in a simple polarimetry configuration at a fixed angle of incidence, consists of maxima whose number and positions depend on the thickness of a thin film. An approximative linear relation between the thin-film thickness and a wavelength of the maximum of the reflectance ratio for a specific angle of incidence is revealed, provided that the wavelength-dependent refractive index of the thin film is known and the substrate is weakly absorbing. The relation permits the calculation of the thickness from the measured spectral reflectance ratio by using one maximum only, as is demonstrated theoretically for a $\mathrm{SiO}_{2}$ thin film on a $\mathrm{Si}$ substrate. The application of this method is demonstrated experimentally for the same thin-film structure with different thicknesses of the $\mathrm{SiO}_{2}$ thin film. The results are compared with those given by the algebraic fitting method, and very good agreement is confirmed.
\end{abstract}

Key words: polarimetry, spectral reflectance ratio, thin film, thickness PACS: 07.60.Fs, 68.55.Jk

* Tel.: +420-597-323-134; fax: +420-597-323-139.

Email address: petr.hlubina@vsb.cz (P. Hlubina). 
Physical thickness of a thin film is a fundamental parameter that can be measured by a number of optical methods. These include ellipsometric measurements performed at a single wavelength and at a fixed angle of incidence [1] or spectral ellipsometry used over a wide wavelength range that provides the results with higher accuracy [2]. Similarly, spectral reflectometry and interferometry applied over a wide wavelength range are used for thin-film and multilayer structure characterization [3-5].

Spectral reflectometry utilizing the reflection of light from a single thin film also plays a crucial role in determining the optical constants that characterize the film and the substrate [6-9]. For weakly absorbing systems, e.g., the thickness of the thin film, the refractive indices, and the extinction coefficients of both the thin film and the substrate are calculated from the envelopes that are tangent to the extrema in the reflectance spectrum and from the wavelengths at which the extrema in the reflectance occur [6]. An efficient modification of the method is available that is based on determining the values of the wavelengths corresponding to the points where the reflectance spectra for several angles of incidence touch the envelopes of extrema [7]. Another method simplifies the calculation of the refractive index of the thin film by constructing a midpoint envelope through the spectrum of normal reflectance [8]. A method of determination of optical parameters of specific thin-film structures from both transmission and reflection measurements is also available [9]. Recently, a new method of determining the thickness of a nonabsorbing thin film on an absorbing substrate from only one extreme of the reflectance spectrum has been presented [10].

Precise measurements of the absolute values of the spectral reflectances or the ellipsometric parameters that can be used to determine the values of the optical parameters of thin films are obviously performed by commercially available spectrometers or ellipsometers. To use a cheaper instrumentation compared to expensive spectral reflectometers and ellipsometers, we propose measurement methods allowing one to characterize thin films employing only the wavelengths of the maxima and minima in the spectral reflectances [7] or the maxima in the ratio between the spectral reflectances of $p$ - and $s$-polarized components measured at oblique incidence of light. The ratio between the spectral reflectances of the two polarization states can be measured in a simple polarimetry configuration [11] using the procedure with only two orientations of an analyzer.

The aim of this paper is to present a simple method to determine the thickness of a nonabsorbing thin film on an absorbing substrate from maxima of the spectral reflectance ratio measured at a fixed angle of incidence. An approximative linear relation between the thin-film thickness and a wavelength of the maximum of the reflectance ratio for a specific angle of incidence is revealed, provided that the wavelength-dependent refractive index of the thin film is 
known and the substrate is weakly absorbing. Using the linear relation, the thin-film thickness can be calculated from the measured spectral reflectance ratio by using one maximum only. This is demonstrated in determining the thicknesses of a $\mathrm{SiO}_{2}$ thin film prepared by thermal oxidation on a $\mathrm{Si}$ wafer. The results are compared with those obtained by the algebraic fitting method [11], and very good agreement is confirmed.

\section{Experimental Method}

Let us consider a simple polarimetry configuration as shown in Fig. 1 that can be used for measuring the wavelength dependence of the ratio between the reflectances of $p$ - and $s$-polarized waves reflected from a thin-film structure. The collimated beam from a white-light source passes through a polarizer oriented $45^{\circ}$ with respect to the plane of incidence so that both $p$ - and $s$-polarized components are generated. Next, these components undergo due to reflection from a thin-film structure at the angle of the incidence $\alpha$ the amplitude and phase changes that are related to the complex reflection coefficients [1]

$$
r_{p, s}(\lambda)=\left[R_{p, s}(\lambda)\right]^{1 / 2} \exp \left[\mathrm{i} \delta_{p, s}(\lambda)\right]
$$

where $R_{p, s}(\lambda)$ and $\delta_{p, s}(\lambda)$ are the wavelength-dependent reflectances and phase changes on reflection for both polarizations, respectively. The procedure for the measurement of the reflectance ratio $R_{p}(\lambda) / R_{s}(\lambda)$, which corresponds to $\tan ^{2} \Psi(\lambda)$, where $\Psi(\lambda)$ is the ellipsometric angle, consists of three steps: first, with the source blocked, the background spectrum $I_{b k g}(\lambda)$ is measured; second, with the analyzer oriented perpendicularly to the plane of the incidence, the reflection spectrum $I_{s}(\lambda)$ for $s$ polarization is measured; and third, with the analyzer oriented parallel to the plane of the incidence, the reflection spectrum $I_{p}(\lambda)$ for $p$ polarization is measured. The reflectance ratio $R_{p}(\lambda) / R_{s}(\lambda)$ of the structure is given by [11]

$$
R_{p}(\lambda) / R_{s}(\lambda)=\left[I_{p}(\lambda)-I_{b k g}(\lambda)\right] /\left[I_{s}(\lambda)-I_{b k g}(\lambda)\right]
$$

Employment of a spectrometer with a linear CCD array detector and with a standard implementation of Eq. (2) in its measurement modes enables one nearly real-time recording of the reflectance ratio $R_{p}(\lambda) / R_{s}(\lambda)$ over a wide wavelength range. 


\section{Theoretical Background}

Let us consider an oblique incidence of light at the angle $\alpha$ from air $\left(n_{0}=1\right)$ on a nonabsorbing thin film with the thickness $d$ and the refractive index $n_{1}(\lambda)$. The film is on a flat absorbing substrate with the complex refractive index $n_{2}(\lambda)-\mathrm{i} \kappa_{2}(\lambda)$, where $\kappa_{2}(\lambda)$ is the wavelength-dependent extinction coefficient. We can write the well-known equation for the spectral reflectances of $p$ - and $s$-polarized components [12]

$$
R_{p, s}(\lambda)=\frac{R_{1 p, s}(\lambda)+R_{2 p, s}(\lambda)+2\left[R_{1 p, s}(\lambda) R_{2 p, s}(\lambda)\right]^{1 / 2} \cos \left[2 \beta(\lambda)+\phi_{p, s}(\lambda)\right]}{1+R_{1 p, s}(\lambda) R_{2 p, s}(\lambda)+2\left[R_{1 p, s}(\lambda) R_{2 p, s}(\lambda)\right]^{1 / 2} \cos \left[2 \beta(\lambda)+\phi_{p, s}(\lambda)\right]},(3)
$$

where the reflectances $R_{1 p, s}(\lambda)$ and $R_{2 p, s}(\lambda)$ are related to the well-known Fresnel formulae [12], and the phase change $\beta(\lambda)$ and the phase shifts $\phi_{p, s}(\lambda)$ are given by

$$
\begin{aligned}
& \beta(\lambda)=\frac{2 \pi}{\lambda} n_{1}(\lambda) d \cos \gamma_{1} \\
& \tan \phi_{p}(\lambda)=\frac{-2 n_{1}(\lambda) \cos \gamma_{2} \kappa_{2}(\lambda) \cos \gamma_{1}}{n_{2}^{2}(\lambda) \cos ^{2} \gamma_{1}-n_{1}^{2}(\lambda) \cos ^{2} \gamma_{2}+\kappa_{2}^{2}(\lambda) \cos ^{2} \gamma_{1}}, \\
& \tan \phi_{s}(\lambda)=\frac{2 n_{1}(\lambda) \cos \gamma_{1} \kappa_{2}(\lambda) \cos \gamma_{2}}{n_{1}^{2}(\lambda) \cos ^{2} \gamma_{1}-n_{2}^{2}(\lambda) \cos ^{2} \gamma_{2}-\kappa_{2}^{2}(\lambda) \cos ^{2} \gamma_{1}},
\end{aligned}
$$

where $\gamma_{1}$ and $\gamma_{2}$ are the angles of refraction at the upper and lower boundaries, respectively.

Next, we can express the ratio $R_{p}(\lambda) / R_{s}(\lambda)$ between the spectral reflectances of $p$ - and $s$-polarized components. Knowing the optical constants of the thinfilm structure, we can construct for the angle of incidence $\alpha$ the envelope tangent to the maxima of the spectral reflectance ratio. When $n_{2}(\lambda)>n_{1}(\lambda)$, the envelope corresponds to the points where

$$
\cos \left[2 \beta(\lambda)+\phi_{s}(\lambda)\right]=-1
$$

The equation contains the relationship between the thin-film thickness and the tangent wavelengths of the reflectance ratio spectrum. To find this relationship from Eq. (7), we have

$$
\frac{4 \pi}{\lambda_{\tan , m}} n_{1}\left(\lambda_{\tan , m}\right) d \cos \gamma_{1}+\phi_{s}\left(\lambda_{\tan , m}\right)=(2 m+1) \pi
$$


where $\lambda_{\tan , m}$ is the tangent wavelength and $m$ is an integer that is referred to as the interference order. Finally, the relation can be rewritten as

$$
d=\frac{\lambda_{\tan , m}}{4 \pi\left[n_{1}^{2}\left(\lambda_{\tan , m}\right)-\sin ^{2} \alpha\right]^{1 / 2}}\left[(2 m+1) \pi-\phi_{s}\left(\lambda_{\tan , m}\right)\right]
$$

In the next subsection we analyze theoretically the wavelength dependence of the spectral reflectance ratio for a thin-film structure of known optical parameters. We also derive an approximative linear relation between the film thickness and a wavelength where the reflectance ratio reaches a maximum.

\subsection{Theoretical Modeling: Examples}

Before deriving the relation that can be used for determination of the thickness of a thin film from the experimental data, we constructed the theoretical spectral reflectance ratio as a function of wavelength, using Eq. (3). Figure 2 shows the spectral reflectances $R_{p}(\lambda), R_{s}(\lambda)$, and the reflectance ratio $R_{p}(\lambda) / R_{s}(\lambda)$ as well, in the wavelength range from 400 to $1000 \mathrm{~nm}$ for the $\mathrm{SiO}_{2}$ thin film of thickness $d=450 \mathrm{~nm}$ on a $\mathrm{Si}$ substrate. The refractive index of the $\mathrm{SiO}_{2}$ was calculated from the standard Sellmeier formula [13]. The refractive index and the extinction coefficient of the Si substrate were taken from [14]. In the considered wavelength range, as seen from Fig. 2, the reflectance $R_{s}(\lambda)$ has two minima that correspond to two interference orders. Similarly, the reflectance ratio $R_{p}(\lambda) / R_{s}(\lambda)$ has two maxima that are easier to identify compared to the minima of the reflectance $R_{s}(\lambda)$. This is due to much greater magnitude of the reflectance ratio peak and its localization in a narrower wavelength range. Moreover, the greater the angle of incidence, the greater magnitude and narrower peak of the reflectance ratio as illustrated in Fig. 3 that shows the wavelength dependence of the spectral reflectance ratio for the same thickness and three angles of the incidence $\alpha=30^{\circ}, 45^{\circ}, 60^{\circ}$.

To demonstrate other interesting properties of the reflectance ratio, we considered two angles of incidence $\alpha=45^{\circ}, 60^{\circ}$ and five thicknesses (ranging from 300 to $500 \mathrm{~nm}$ ) of the $\mathrm{SiO}_{2}$ thin film on the Si substrate. As seen from Figs. 4 and 5 , first, the number of the maxima and their positions are dependent on the thin-film thickness. For the film thickness $d=350 \mathrm{~nm}$, e.g., a single maximum can be resolved in the considered wavelength range. Furthermore, the longer the wavelength, the greater the magnitude of the maximum of the reflectance ratio. In Figs. 4 and 5 are also shown the approximate envelopes of the theoretical spectral reflectance ratio constructed from the spectral reflectances $R_{p}(\lambda)$ and $R_{s}(\lambda)$ given by Eq. (3) when an assumption $\phi_{p}(\lambda) \approx \phi_{s}(\lambda)$ corresponding to a weakly absorbing substrate [7] is included. For these phase shifts, we 
obtain from Eqs. (3) and (7) the envelope equations

$$
E_{ \pm}(\lambda)=R_{p}^{E_{ \pm}}(\lambda) / R_{s}^{E}(\lambda)
$$

where

$$
R_{p}^{E_{ \pm}}(\lambda)=\frac{R_{1 p}(\lambda)+R_{2 p}(\lambda) \pm 2\left[R_{1 p}(\lambda) R_{2 p}(\lambda)\right]^{1 / 2}}{1+R_{1 p}(\lambda) R_{2 p}(\lambda) \pm 2\left[R_{1 p}(\lambda) R_{2 p}(\lambda)\right]^{1 / 2}}
$$

and

$$
R_{s}^{E}(\lambda)=\frac{R_{1 s}(\lambda)+R_{2 s}(\lambda)-2\left[R_{1 s}(\lambda) R_{2 s}(\lambda)\right]^{1 / 2}}{1+R_{1 s}(\lambda) R_{2 s}(\lambda)-2\left[R_{1 s}(\lambda) R_{2 s}(\lambda)\right]^{1 / 2}} .
$$

For the angle of incidence $\alpha=45^{\circ}$ the approximate envelope is given by $R_{p}^{E_{-}}(\lambda)$, whereas for the angle of incidence $\alpha=60^{\circ}$ the approximate envelope is given by $R_{p}^{E_{+}}(\lambda)$. If light absorption is small, the true envelopes are the same as the approximate ones and the tangent wavelengths $\lambda_{\tan , m}$ are practically identical to the wavelengths $\lambda_{\max , m}$ of the maxima, as can be seen from Fig. 4 at, e.g., longer wavelengths. However, when absorption of the substrate becomes significant (at shorter wavelengths), the true envelopes differ from the approximate ones, but they touch the spectral reflectance ratio at wavelengths $\lambda_{\tan , m}$, which are shifted left from $\lambda_{\max , m}$. Generally, this shows that the use of $\lambda_{\tan , m}$ instead of $\lambda_{\max , m}$ is more accurate.

However, from the practical point of view, it is easier to determine $\lambda_{\max , m}$ than $\lambda_{\tan , m}$. This is especially true for the curves of the reflectance ratio with a single maximum whose envelope cannot be constructed. Thus, the next step is the construction of the approximative function $d=f\left(\lambda_{\max , m}\right)$ derived from Eq. (9) when we once again assume a weakly absorbing substrate, i.e., $\phi_{s}(\lambda)$ can be neglected [7]. This dependence is illustrated in Fig. 6 for three angles of the incidence $\alpha=30^{\circ}, 45^{\circ}, 60^{\circ}$. According to Fig. 6 , it is evident that the functions are linear (the correlation coefficients of the linear fits are 1) and can be expressed as

$$
d=A+B \lambda_{\max , m},
$$

where the characteristic parameters $A$ and $B$ are connected, as results from Eq. (9), with the wavelength-dependent refractive index $n_{1}(\lambda)$ of the thin film, the angle of the incidence $\alpha$ and interference order $m$ only. Parameters $A$ and $B$ of Eq. (13) obtained for the $\mathrm{SiO}_{2}$ thin film in a wavelength range from 450 to $900 \mathrm{~nm}$ for all three angles of the incidence are listed in Table 1, which also shows the errors $\delta A$ and $\delta B$ of the parameters resulting from the linear fits. 
It is evident from Eq. (13) that, if the wavelength of the maximum $\lambda_{\max , m}$ and the interference order $m$ are known for the given angle of the incidence $\alpha$, we can determine the thin-film thickness $d$ through one spectral reflectance ratio measurement around one maximum only. The wavelength of the maximum

$\lambda_{\max , m}$ can be determined easily thanks to sufficiently pronounced maximum of the reflectance ratio of the thin-film structure. However, because of a possible overlap of the maxima corresponding to different thicknesses and interference orders (see Figs. 4 and 5), an additional estimate of the thin-film thickness by a different technique (e.g., normal incidence reflectometry) is needed. Moreover, as illustrates Fig. 6, the measurements performed in the visible range are not applicable to the thicknesses smaller than $200 \mathrm{~nm}$. Table 1 shows examples of the thicknesses $d$ determined for three angles of incidence and their comparison with the theoretical thickness $d=450 \mathrm{~nm}$. According to Table 1, the differences between the determined and the theoretical thicknesses were found to be less than $0.3 \mathrm{~nm}$. This error, which is affected by the true absorption of the substrate, is larger in a short-wavelength range.

It should be stressed here that this procedure is much simpler compared with that used in normal incidence reflectometry where the linear relation between the thin-film thickness $d_{\tan }$ and the tangent wavelength $\lambda_{\tan , m}$ is used [10]. The tangent wavelength $\lambda_{\tan , m}$ is determined using the minimum deviation between the reflectance of the thin-film structure and the envelope of the reflectance around the specific reflection extreme (see a procedure in [7]). Our new method uses the simplification of Eq. (9) under the condition of a weakly absorbing substrate which leads to Eq. (13). In this case $\lambda_{\tan , m} \approx \lambda_{\max , m}$ and the method is restricted to thin films whose the refractive index $n_{1}(\lambda)$ is known.

\section{Experimental setup}

The experimental setup used for measurement of the reflectance ratio $R_{p}(\lambda) / R_{s}(\lambda)$ of a thin-film structure is shown schematically in Fig. 1 and it consists of a white-light source: a halogen lamp HL-2000 (Ocean Optics) with launching optics, an optical fiber and a collimating lens, Glan Taylor calcite polarizer and analyzer (Thorlabs), a thin-film structure, a microscope objective, micropositioners, a read optical fiber, a fiber-optic spectrometer S2000 (Ocean Optics), an A/D converter and a personal computer. The spectrometer has a spectral operation range from 350 to $1000 \mathrm{~nm}$ and it can be operated in a transmission mode [15] to measure the reflectance ratio $R_{p}(\lambda) / R_{s}(\lambda)$ according to Eq. (2).

We demonstrate the ability of our new method in measuring the reflectance ratio $R_{p}(\lambda) / R_{s}(\lambda)$ for three samples of $\mathrm{SiO}_{2}$ thin films on a Si substrate using a light beam of $4 \mathrm{~mm}$ in diameter. The $\mathrm{SiO}_{2}$ thin films on the Si wafer were prepared by using a dry oxidation process described by the so-called 
Deal-Grove model [16]. Single-crystal silicon wafers from ON Semiconductor, Czech Republic, were characterized by subsequent parameters: diameter $(100 \pm 0.5) \mathrm{mm}$, orientation (111), B doped type P, thickness $(381 \pm 25) \mu \mathrm{m}$ and resistivity $(0.008 \div 0.009) \Omega \mathrm{cm}$. Before the oxidation, the wafers were cut into $40 \times 40 \mathrm{~mm}$ squares, cleaned by standard methods and then annealed in a furnace at $1200^{\circ} \mathrm{C}$. Three annealing times were selected in order to prepare $\mathrm{SiO}_{2}$ thin film with three thicknesses ranging approximately from 300 to $450 \mathrm{~nm}$.

\section{Experimental results and discussion}

The wavelength dependence of the reflectance ratio $R_{p}(\lambda) / R_{s}(\lambda)$ was measured by the three-step procedure presented above for three angles of the incidence $\alpha=30^{\circ}, 45^{\circ}, 60^{\circ}$. The reflectance ratios measured at the angle of the incidence $\alpha=45^{\circ}$ for all three samples are shown in Fig. 7. The ratio $R_{p}(\lambda) / R_{s}(\lambda)$ measured for sample 3 includes two maxima in a wavelength range from 450 to 900 $\mathrm{nm}$, whereas the remaining samples include only one maximum. First, using our new approach, the approximative linear function given by Eq. (13) was utilized to determine the thin-film thickness $d$ from the position $\lambda_{\max , m}$ of a maximum in the measured spectral reflectance ratio. From the experimental reflectance ratios of the samples in the measured wavelength range we have chosen only maxima with interference order $m=1$ (those at longer wavelengths, as can be seen from Fig. 7). In Table 2 are listed the values $\lambda_{\max , 1}$ together with the thicknesses $d$ corresponding to Eq. (13). The values $\lambda_{\max , 1}$ were determined as precise as possible from the positions of the maxima of the smoothed upper parts of the reflectance ratio curves. If we assume that the precision in determining the wavelengths is in the best case $0.32 \mathrm{~nm}$ (wavelength distance of two adjacent pixels of spectrometer S2000), the error in measuring the thicknesses is $0.2 \mathrm{~nm}$. However, the positions of the maxima for the flat reflectance ratio curves (angles of the incidence $\alpha=30^{\circ}, 45^{\circ}$ ) are determined with lower precisions so that the error in measuring the thicknesses exceeds $1 \mathrm{~nm}$. Table also presents the experimental data for the remaining angles of the incidence (sample 3) and the data for samples 1 and 2. As an example, Fig. 8 shows the reflectance ratios measured for sample 2 at all three angles of the incidence.

Second, using a standard approach with the Levenberg-Marquardt least-squares algorithm [17], the measured reflectance ratio $R_{p}(\lambda) / R_{s}(\lambda)$ was fitted to the theoretical one evaluated by means of Eq. (3) in order to obtain the thin-film thickness $d_{f i t}$. The theoretical reflectance ratio $R_{p}(\lambda) / R_{s}(\lambda)$ was calculated in a wavelength range from 450 to $900 \mathrm{~nm}$ using the refractive index of the $\mathrm{SiO}_{2}$ given by the standard Sellmeier formula [13] and the refractive index and the extinction coefficient of silicon substrate taken from [14]. The theoretical dependences for the angle of the incidence $\alpha=45^{\circ}$ and all three samples are 
shown in Fig. 7 by the dashed curves. They demonstrate that positions of the maxima in the measured reflectance ratios agree well with theory. The difference in absolute values is apparent for sample 2, which is probably caused by nonideal alignment of the analyzer with respect to the detection part in the polarimetry configuration. Similarly, Fig. 8 shows by the dashed curves the theoretical reflectance ratios corresponding to sample 2 and all three angles of the incidence. Figures 7 and 8 demonstrate a good agreement between the theory and experiment. The thicknesses $d_{f i t}$ of the $\mathrm{SiO}_{2}$ thin films determined by this procedure (from the measured reflectance ratios) for all three samples and angles of incidence are listed in Table 2.

The efficiency of the first approach (our new method) is clearly seen from Table 2 , which demonstrates a very good agreement between the thicknesses determined by using Eq. (13) and those determined by using the second approach (the algebraic fitting method).

\section{Conclusions}

A simple method to determine the thickness of the nonabsorbing thin film on the absorbing substrate has been presented. The linear relation between the thin-film thickness and the wavelength of a maximum of the spectral reflectance ratio measurable in a simple polarimetry configuration at a specific angle of incidence was revealed. This relation, which was derived under the condition of a weakly absorbing substrate, enabled the calculation of thin-film thickness from one measurement of the spectral reflectance ratio provided that the wavelength-dependent refractive index of the thin film is known. This fact of course limits the applicability of the method. The thin-film thickness determination from one maximum of the spectral reflectance ratio was demonstrated theoretically for a $\mathrm{SiO}_{2}$ thin film on a Si substrate. The new procedure was also demonstrated experimentally for the same thin-film structure with different thicknesses of the $\mathrm{SiO}_{2}$ thin film. The obtained thicknesses were compared with those resulting from the fit of the measured spectral reflectance ratios by using a least-squares algorithm, and good agreement was confirmed.

This simple and cost effective method could be well applied also in other cases; e.g. when the spectral reflectance ratios indicate only one clear maximum due to a too narrow wavelength interval of the recording. The method could be also used for measurement of small thin-film thickness changes.

Acknowledgements The research has been partially supported by the Ministry of Education, Youth and Sports of the Czech Republic through grant MSM6198910016 and by the regional grant CZ.1.05/2.1.00/01.0040. 


\section{References}

[1] Azzam RMA, Bashara NM. Ellipsometry and Polarized Light. Amsterdam: North-Holland; 1987.

[2] Jellison GEJr. The calculation of thin film parameters from spectroscopic ellipsometry data. Thin Solid Films 1996;290-291:40-5.

[3] Hlubina P, Luňáček J, Ciprian D, Chlebus R. Spectral interferometry and reflectometry used to measure thin films. Appl Phys B 2008;92:203-7.

[4] Case WE. Algebraic method for extracting thin film parameters from spectrophotometer measurements. Appl Opt 1983;22:1832-6.

[5] Merklein TM. High-resolution measurement of multilayer structures. Appl Opt 1990;29:505-11.

[6] Rusli, Amaratunga GAJ. Determination of the optical constants and thickness of thin films on slightly absorbing substrates. Appl Opt 1995;34:7914-24.

[7] Ohlídal I, Franta D,Ohlídal M, Navrátil K, Optical Characterization of nonabsorbing and weakly absorbing thin films with the wavelength related to extrema in spectral reflectances. Appl Opt 2001;40:5711-7.

[8] Humphrey S. Direct calculation of the optical constants for a thin film using a midpoint envelope. Appl Opt 2007;46:4660-6.

[9] Stenzel O, Hopfe V, Klobes P. Determination of optical parameters for amorphous thin film materials on semitransparent substrates from transmittance and reflectance measurements. J Phys D 1991;24:2088-94.

[10] Luňáček J,Hlubina P, Luňáčková M Simple method for determination of the thickness of a nonabsorbing thin film using spectral reflectance measurement. Appl Opt 2009;48:985-9.

[11] Hlubina P, Lunacek J, Ciprian D. Spectral interferometric technique to measure ellipsometric phase of a thin-film structure. Opt Lett 2009;34:2661-3.

[12] Born M,Wolf E. Principles of Optics. Cambridge: Cambridge University Press; 1999.

[13] Hlubina P, Ciprian D, Luňáček J, Lesňák M. Dispersive white-light spectral interferometry with absolute phase retrieval to measure thin film. Opt Express $2006 ; 14: 7678-85$.

[14] Palik ED. Handbook of Optical Constants of Solids. Orlando: Academic Press; 1995.

[15] Operating Manual and Users Guide. S2000 Miniature Fiber Optic Spectrometers and Accessories. Dunedin: Ocean Optics; 2000.

[16] Plummer JD, Deal MD, Griffin PB. Silicon VLSI Technology Fundamentals, Practice and Modeling. Upper Saddle River: Prentice Hall; 2000. 
[17] Optimization Toolbox for Use with MATLAB. Massachusetts: MathWorks; 2000 . 
Table 1

Parameters $A$ and $B$ for the angles of incidence $\alpha$ and the $\mathrm{SiO}_{2}$ thin film on a Si substrate with the wavelengths $\lambda_{\max , m}$ and the thicknesses $d$ corresponding to Eq. (13)

\begin{tabular}{ccccccc}
$\alpha\left(^{\circ}\right)$ & $A(\mathrm{~nm})$ & $\delta A(\mathrm{~nm})$ & $B$ & $\delta B$ & $\lambda_{\max , 1}(\mathrm{~nm})$ & $d(\mathrm{~nm})$ \\
\hline 30 & -4.5045 & \pm 0.0119 & 0.54988 & \pm 0.00002 & 827.01 & 450.3 \\
45 & -5.5590 & \pm 0.0146 & 0.59098 & \pm 0.00002 & 771.31 & 450.3 \\
60 & -7.1025 & \pm 0.0185 & 0.64297 & \pm 0.00003 & 711.23 & 450.2 \\
\hline
\end{tabular}

Table 2

Oxidation times $T$, angles of incidence $\alpha$, wavelengths $\lambda_{\max , m}$, thicknesses $d$ and $d_{f i t}$ of the $\mathrm{SiO}_{2}$ thin films

\begin{tabular}{|c|c|c|c|c|c|}
\hline Sample & $T(\min )$ & $\alpha\left(^{\circ}\right)$ & $\lambda_{\max , 1}(\mathrm{~nm})$ & $d(\mathrm{~nm})$ & $d_{f i t}(\mathrm{~nm})$ \\
\hline \multirow{3}{*}{1} & \multirow{3}{*}{122} & 30 & 530.89 & 287.4 & 286.6 \\
\hline & & 45 & 497.62 & 288.5 & 287.7 \\
\hline & & 60 & 459.83 & 288.6 & 286.5 \\
\hline \multirow{3}{*}{2} & \multirow{3}{*}{212} & 30 & 624.57 & 338.9 & 338.6 \\
\hline & & 45 & 580.97 & 337.8 & 337.5 \\
\hline & & 60 & 537.03 & 338.2 & 337.3 \\
\hline \multirow{3}{*}{3} & \multirow{3}{*}{326} & 30 & 830.02 & 451.9 & 450.8 \\
\hline & & 45 & 775.03 & 452.5 & 451.9 \\
\hline & & 60 & 715.05 & 452.7 & 452.4 \\
\hline
\end{tabular}




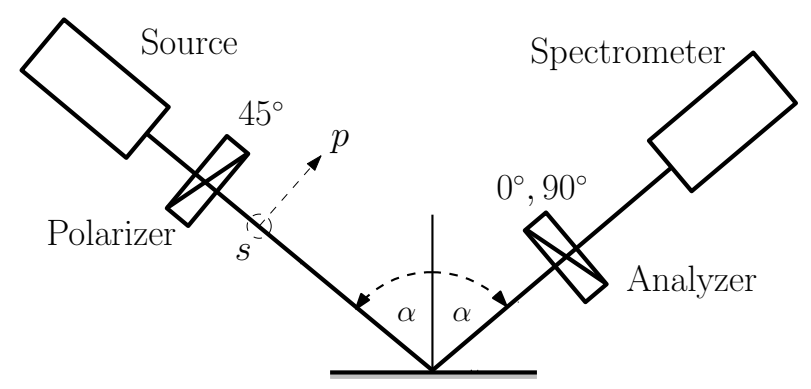

Thin-film structure

Fig. 1. Experimental setup for measuring the wavelength dependence of the reflectance ratio $R_{p}(\lambda) / R_{s}(\lambda)$ of a thin-film structure for the angle of incidence $\alpha$.

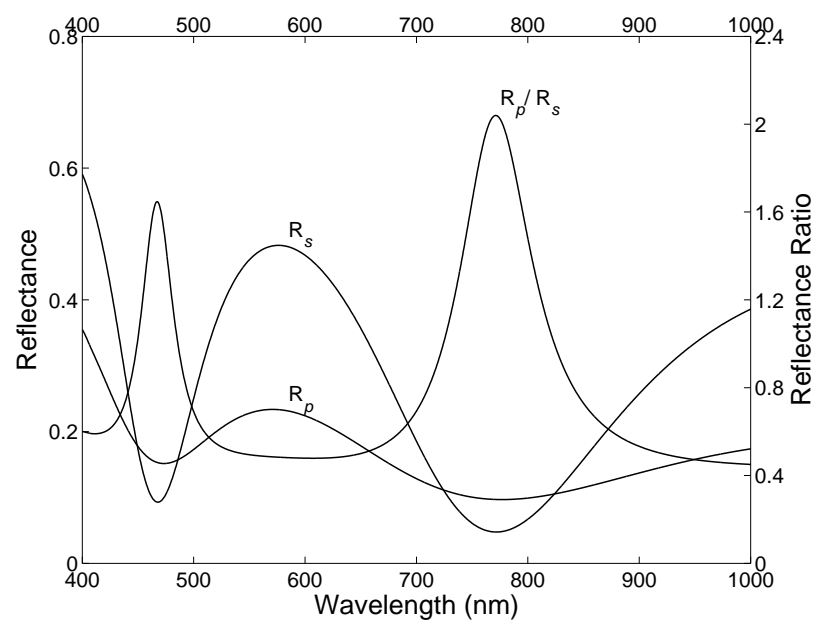

Fig. 2. Theoretical wavelength dependences of the reflectances $R_{p}(\lambda), R_{s}(\lambda)$ and the reflectance ratio $R_{p}(\lambda) / R_{s}(\lambda)$ for $\alpha=45^{\circ}$ and the $\mathrm{SiO}_{2}$ thin film of the thickness $d=450 \mathrm{~nm}$ on a Si substrate. 


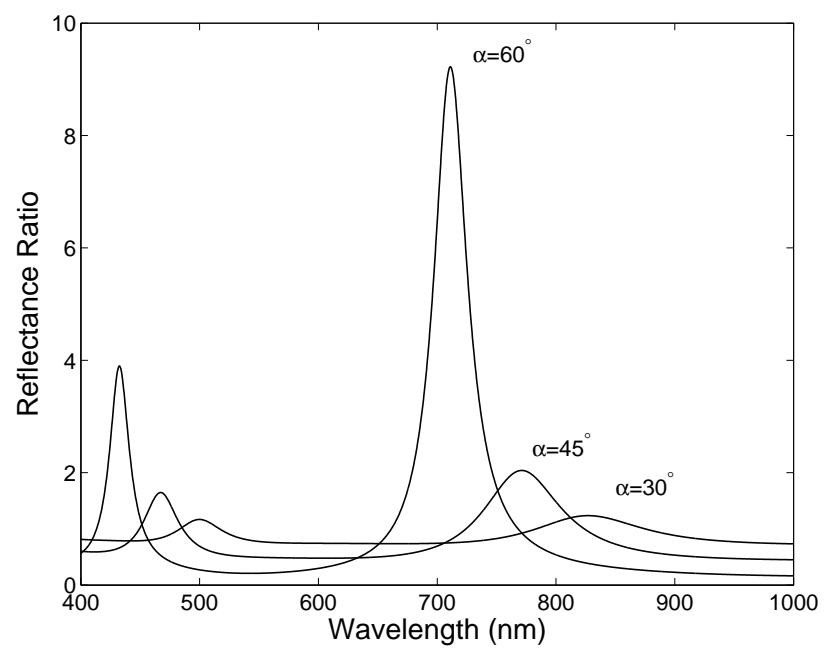

Fig. 3. Theoretical wavelength dependences of the reflectance ratio $R_{p}(\lambda) / R_{s}(\lambda)$ for $\alpha=30^{\circ}, 45^{\circ}, 60^{\circ}$ and the $\mathrm{SiO}_{2}$ thin film of the thickness $d=450 \mathrm{~nm}$ on a Si substrate.

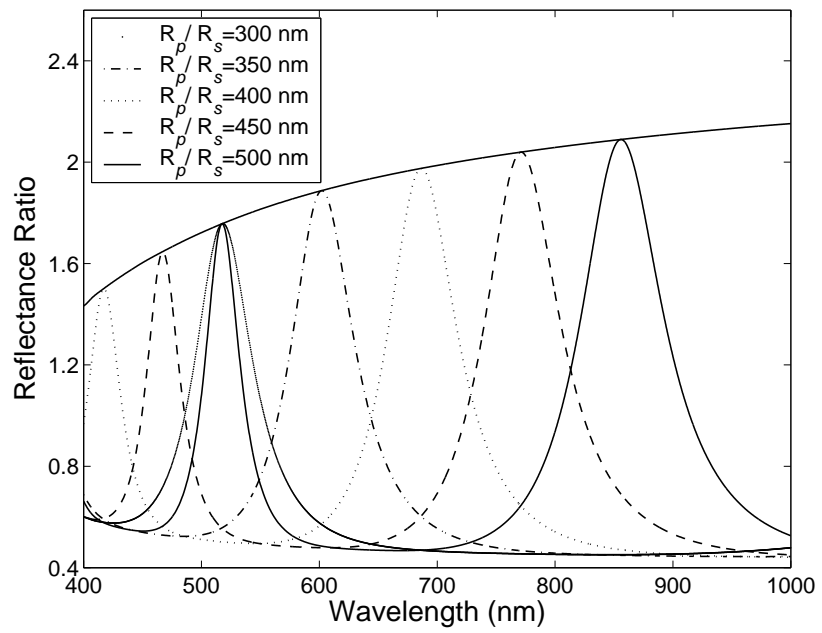

Fig. 4. Theoretical wavelength dependences of the reflectance ratio $R_{p}(\lambda) / R_{s}(\lambda)$ for $\alpha=45^{\circ}$ and the $\mathrm{SiO}_{2}$ thin film of the thicknesses $d=300,350,400,450,500 \mathrm{~nm}$ on a Si substrate. 


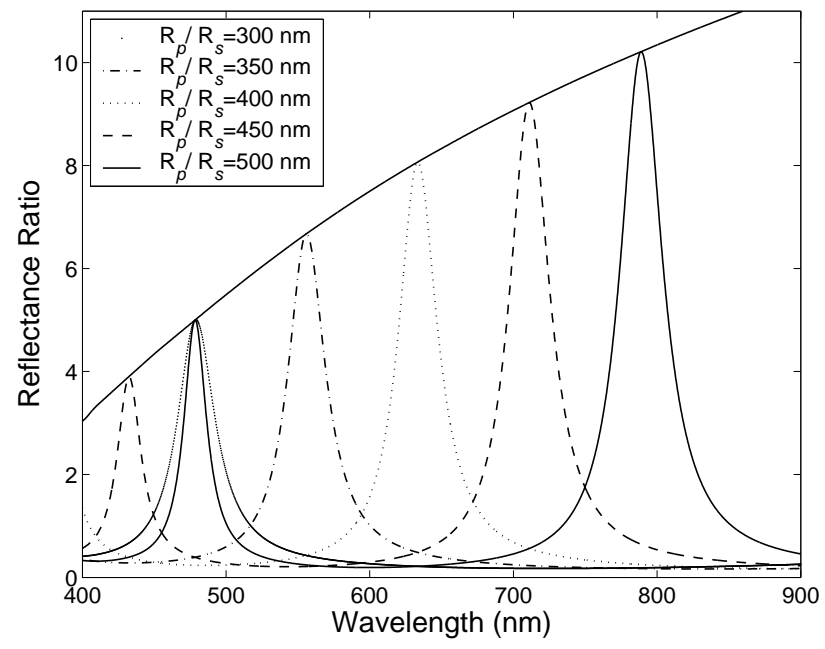

Fig. 5. Theoretical wavelength dependences of the reflectance ratio $R_{p}(\lambda) / R_{s}(\lambda)$ for $\alpha=60^{\circ}$ and the $\mathrm{SiO}_{2}$ thin film of thicknesses $d=300,350,400,450,500 \mathrm{~nm}$ on a $\mathrm{Si}$ substrate.

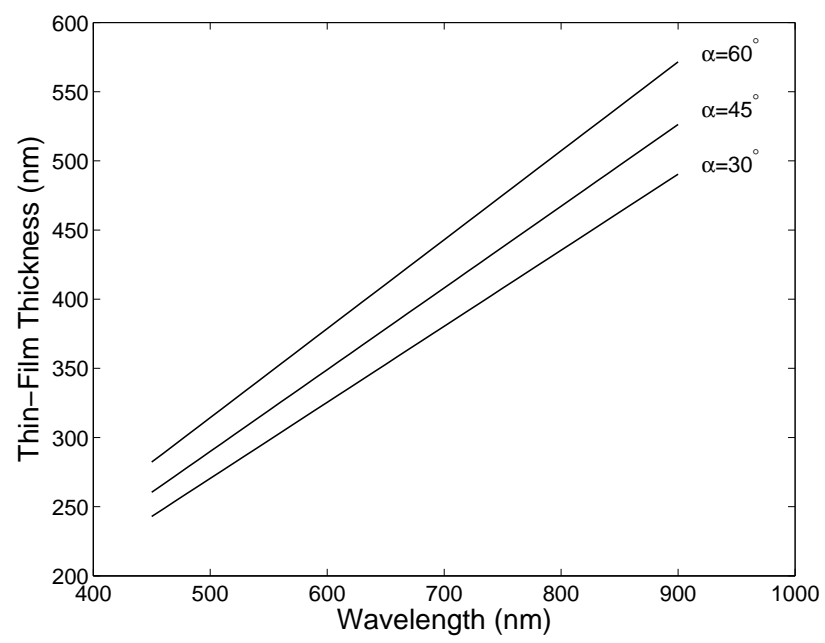

Fig. 6. Approximative linear dependences of the thin film thickness on the wavelength of a maximum of the reflectance ratio $R_{p}(\lambda) / R_{s}(\lambda)$ for $\alpha=30^{\circ}, 45^{\circ}, 60^{\circ}$ and the $\mathrm{SiO}_{2}$ thin film on a $\mathrm{Si}$ substrate. 


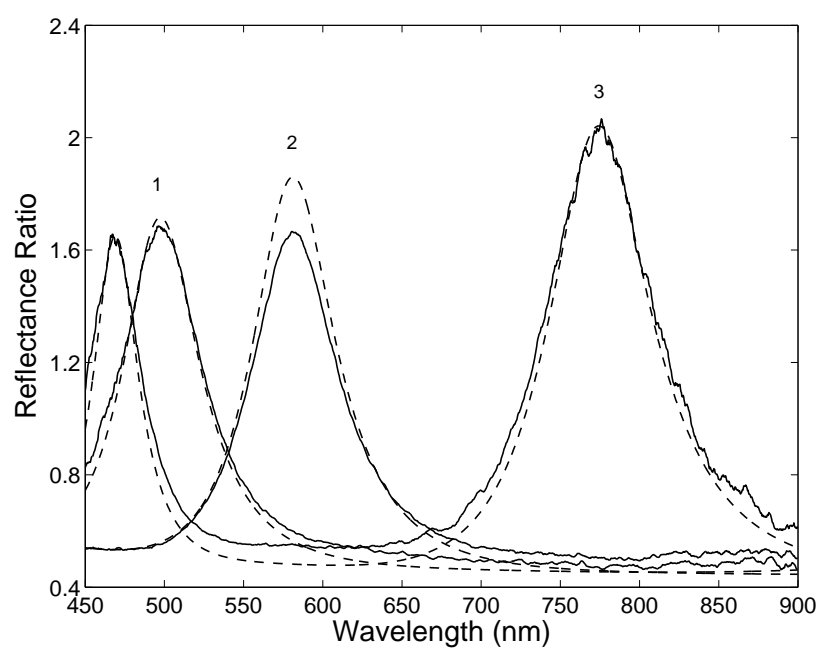

Fig. 7. Measured and theoretical (dashed) wavelength dependences of the reflectance ratio $R_{p}(\lambda) / R_{s}(\lambda)$ for $\alpha=45^{\circ}$ and three different samples of the $\mathrm{SiO}_{2}$ thin film on a Si substrate.

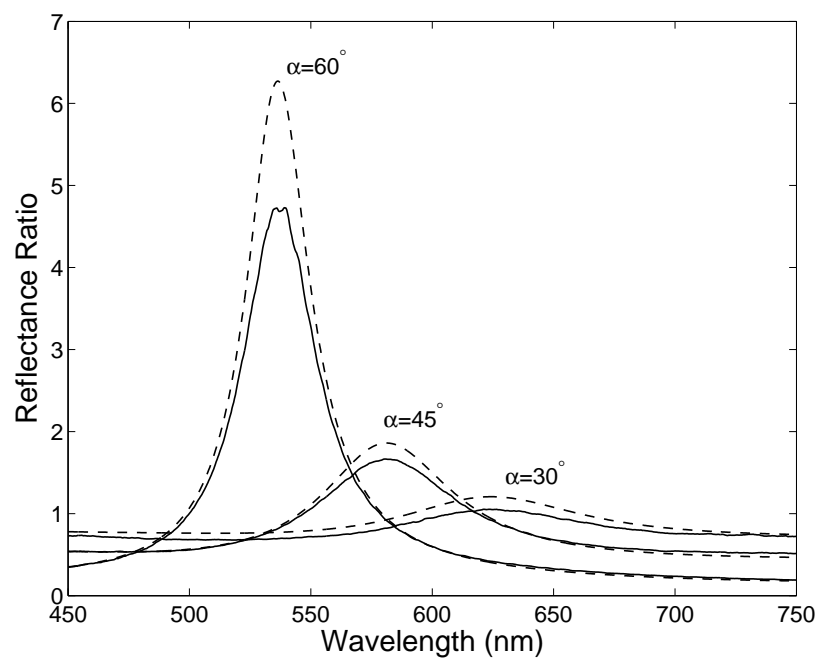

Fig. 8. Measured and theoretical (dashed) wavelength dependences of the reflectance ratio $R_{p}(\lambda) / R_{s}(\lambda)$ for $\alpha=30^{\circ}, 45^{\circ}, 60^{\circ}$ and sample 2 of the $\mathrm{SiO}_{2}$ thin film on a $\mathrm{Si}$ substrate. 